УДК 519.633

\title{
ВЕКТОРНЫЕ АДДИТИВНЫЕ СХЕМЫ ДЛЯ НЕКОТОРЫХ КЛАССОВ УРАВНЕНИЙ ГИПЕРБОЛИЧЕСКОГО ТИПА
}

\author{
М. Х. Шхануков-Лафишев, С. М. Архестова, \\ М. Б. Тхамоков
}

Посвящается шестидесятилетию
Анатолия Георгиевича Кусраева

\begin{abstract}
В работе построены векторно-аддитивные схемы для некоторых классов уравнений гиперболического типа, возникающих в теории влагопереноса и в теории волн в релаксирующих средах. Доказаны устойчивость и сходимость разностных схем в классе достаточно гладких решений рассматриваемых уравнений.
\end{abstract}

Ключевые слова: волновое уравнение, уравнение влагопереноса, устойчивость, сходимость, априорная оценка, вязкость, теплопроводность, погрешность аппроксимации.

\section{Введение}

Для численного решения многомерных задач математической физики используются различные классы аддитивных схем (схем расщепления [1-2]). В работах [3-4] для решения многомерных задач предложен новый класс безусловно устойчивых схем переменных направлений полной аппроксимации. Устойчивость и сходимость таких схем исследуется на основе получения соответствующих априорных оценок. В работе [5] основные принципы построения векторных аддитивных схем на основе операторно-разностных подходов применены к абстрактной задаче Коши. В работе [6] на основе общей теории устойчивости разностных схем $[1,7]$ проводится исследование векторно-аддитивных схем с самосопряженными операторами. В работе [8] на основе общей теории устойчивости операторно-разностных схем А. А. Самарского проводится исследование векторных аддитивных схем для общих эволюционных уравнений первого порядка с несамосопряженными операторами. В [9] на основе принципа регуляризации построены новые аддитивные операторно-разностные схемы полной аппроксимации. В [10] исследуется устойчивость в произвольных нормах векторной аддитивной схемы. Показано, что устойчивость имеет место при условии, что устойчивыми являются чисто неявные схемы для отдельных компонент.

В [11] построены регуляризованные аддитивные операторно-разностные схемы для эволюционных задач без предположения о перестановочности регуляризующего оператора и оператора задачи, отмечены возможности обобщения предложенных регуляризованных аддитивных схем на задачи с несамосопряженными операторами и уравнения второго порядка.

(C) 2013 Шхануков-Лафишев М. Х., Архестова С. М., Тхамоков М. Б. 
В данной работе проводится исследование векторных аддитивных схем для псевдопараболических уравнений (уравнение влагопереноса Аллера [12, с. 137]), волнового уравнения в релаксирующих средах (см. $[13$, с. 84]) и волнового уравнения с учетом вязкости и теплопроводности (см. [13, с. 22]).

Существование и единственность решений, рассматриваемых здесь краевых задач, были исследованы в работах одного из авторов $[14,15]$.

В уравнениях, рассматриваемых в этой работе, содержатся смешанные производные $u_{x_{\alpha} x_{\alpha} t}, u_{x_{\alpha} x_{\alpha} t t}, \alpha=\overline{1, p}$. В этом случае не удается построить разностные схемы на основе понятия суммарной аппроксимации. Например, доказательство сходимости локальноодномерной схемы проводится (см. [1, с. 528]) с помощью введения $\eta_{(\alpha)}$ в задачу для погрешности $z_{(\alpha)}=v_{(\alpha)}+\eta_{(\alpha)}$. В нашем случае в правую часть уравнения для $v_{(\alpha)}=v^{j+\frac{\alpha}{p}}$, $\widetilde{\psi_{\alpha}}$ вмешиваются слагаемые $\Lambda_{\alpha} \eta_{\alpha \bar{t}}=O(1), \Lambda_{\alpha} y=y_{\bar{x}_{\alpha} x_{\alpha}}$, т. е. $\widetilde{\psi_{\alpha}}=\Lambda_{\alpha} \eta_{(\alpha)}+\Lambda_{\alpha} \eta_{\alpha \bar{t}}+\psi_{\alpha}^{*}$, где $\Lambda_{\alpha} \eta_{(\alpha)}=O(\tau), \psi_{\alpha}^{*}=O\left(h_{\alpha}^{2}+\tau\right)$. При $p=2$ можно получить априорную оценку не вводя $\eta_{(\alpha)}$ (см. [7, с. 336]), но только для уравнений, не содержащих смешанные производные. Аддитивные схемы полной аппроксимации [3-10] оказываются более приемлемыми для численного решения рассматриваемых здесь задач.

\section{1. Волновое уравнение в неидеальной среде с учетом вязкости и теплопроводности}

В цилиндре $Q_{T}=G \times(0, T]$, основанием которого является $p$-мерный параллелепипед $G=\left\{x=\left(x_{1}, x_{2}, \ldots, x_{p}\right): 0<x_{\alpha}<l_{\alpha}, \alpha=\overline{1, p}\right\}$, рассмотрим задачу

$$
\begin{gathered}
\frac{\partial^{2} u}{\partial t^{2}}+L u=f(x, t), \quad L u=\sum_{\alpha=1}^{p} L_{\alpha} u, \quad(x, t) \in Q_{T}, \\
\left.u\right|_{S_{T}}=0, \quad u(x, 0)=u_{0}(x), \quad u_{t}(x, 0)=u_{1}(x),
\end{gathered}
$$

где $L_{\alpha} u=-\left[\frac{\partial}{\partial x_{\alpha}}\left(k_{\alpha}(x) \frac{\partial u}{\partial x_{\alpha}}\right)+\frac{\partial}{\partial t} \frac{\partial}{\partial x_{\alpha}}\left(k_{\alpha}(x) \frac{\partial u}{\partial x}\right)\right], \bar{G}=G+\Gamma, S_{T}=\Gamma \times[0, T]-$ боковая поверхность цилиндра $Q_{T}, 0<c_{1} \leqslant k_{\alpha}(x) \leqslant c_{2}, \alpha=\overline{1, p}, k_{\alpha}(x), f(x, t)$ таковы, что решение задачи (1.1)-(1.2) существует, единственно и обладает требуемой гладкостью.

Уравнение вида (1.1) возникает в теории волн (см. [12, с. 85]). Оно учитывает потери энергии, связанные с вязкостью и теплопроводностью. Третий член в левой части уравнения (1.1) описывает затухание звука из-за вязкости и теплопроводности, обычно перед ним стоит диссипативный коэффициент. Ради простоты мы положим его равным единице.

Задачу (1.1)-(1.2) перепишем в виде абстрактной задачи Коши

$$
\begin{gathered}
\frac{d^{2} u}{d t^{2}}+A u+A \frac{d u}{d t}=f(t), \quad A=\sum_{\alpha=1}^{p} A_{\alpha}, \quad A_{\alpha}=-\frac{\partial}{\partial x_{\alpha}}\left(k_{\alpha}(x) \frac{\partial}{\partial x_{\alpha}}\right), \\
u(0)=u_{0}, \quad u_{t}(0)=u_{1},
\end{gathered}
$$

с линейным самосопряженным положительно-определенным оператором $A: H \rightarrow H$, действующим в некотором гильбертовом пространстве $H$ со скалярным произведением $(u, v)=\int_{G} u v d x, A_{\alpha}$ - положительно-определенные операторы в $H$.

Краевые условия учитываются включением $u(t) \in D(A)$, где $D(A)$ - область определения оператора $A$. Вместо одного скалярного решения $u(t)$ вводится вектор решений 
$U(t)=\left(u_{1}(t), u_{2}(t), \ldots, u_{p}(t)\right)$ (см. [3-10]). Вместо исходной задачи (1.3)-(1.4) запишем систему однотипных подзадач:

$$
\begin{aligned}
& \frac{d^{2} u_{\alpha}}{d t^{2}}+\sum_{\beta=1}^{p} A_{\beta} u_{\beta}+\sum_{\beta=1}^{p} A_{\beta} u_{\beta t}=f(t), \\
& u_{\alpha}(0)=u_{0}, \quad u_{\alpha t}(0)=u_{1}, \quad \alpha=\overline{1, p} .
\end{aligned}
$$

\section{2. Устойчивость по правой части и начальным данным решения вспомогательной системы}

Заметим, что дифференциальные операторы $A_{\alpha}, \alpha=\overline{1, p}$, порожденные дифференциальными выражениями $L_{\alpha}^{0} u=-\frac{\partial}{\partial x_{\alpha}}\left(k_{\alpha}(x) \frac{\partial u}{\partial x_{\alpha}}\right)$ и условиями

$$
\begin{gathered}
u\left(x_{1}, \ldots, x_{\alpha-1}, 0, x_{\alpha+1}, \ldots, x_{p}, t\right)=u\left(x_{1}, \ldots, x_{\alpha-1}, l_{\alpha}, x_{\alpha+1}, \ldots, x_{p}, t\right)=0, \quad t \in[0, T], \\
k_{\alpha}(x) \geqslant c_{1}>0, \quad \alpha=\overline{1, p}
\end{gathered}
$$

являются постоянными (не зависят от $t$ ), самосопряженными и положительно определенными.

Утверждение. Задача (1.5)-(1.6), полученная в результате перехода от скалярного решения задачи (1.3)-(1.4) к вектор-решению, поставлена корректно, причем $u_{\alpha}(t)=$ $u(t), \alpha=\overline{1, p}$, для $t \in[0, T]$ и справедлива оценка

$$
\begin{gathered}
\sum_{\alpha=1}^{p}\left\|u_{\alpha x_{\alpha} t}\right\|^{2}+\left\|\sum_{\alpha=1}^{p} A_{\alpha} u_{\alpha}\right\|^{2} \leqslant M\left(\|f\|_{2 Q_{t}}^{2}+\left\|A u_{0}\right\|^{2}+\sum_{\alpha=1}^{p}\left\|u_{1 x_{\alpha}}\right\|^{2}\right), \\
\|f\|_{2, Q_{t}}^{2}=\int_{0}^{t}\|f\|^{2} d \tau, \quad\|f\|^{2}=\int_{G} f^{2}(x) d x .
\end{gathered}
$$

$\triangleleft$ Пусть $z_{\alpha}=u_{\alpha}-u$. Подставляя $u_{\alpha}=z_{\alpha}+u$ в уравнение (1.5), находим

$$
\begin{gathered}
\frac{\partial^{2} z_{\alpha}}{\partial t^{2}}+\sum_{\beta=1}^{p} A_{\beta} z_{\beta}+\sum_{\beta=1}^{p} A_{\beta} z_{\beta t}=0, \quad \alpha=\overline{1, p}, \\
z_{\alpha}(0)=z_{\alpha t}(0)=0 .
\end{gathered}
$$

Каждое из уравнений (2.2) умножим скалярно на $A_{\alpha} \frac{\partial z_{\alpha}}{\partial t}$ и просуммируем их по $\alpha=$ $\overline{1, p}$. Тогда получим

$$
\sum_{\alpha=1}^{p}\left(\frac{\partial^{2} z_{\alpha}}{\partial t^{2}}, A_{\alpha} \frac{\partial z_{\alpha}}{\partial t}\right)+\frac{1}{2} \frac{\partial}{\partial t}\left\|\sum_{\alpha=1}^{p} A_{\alpha} z_{\alpha}\right\|^{2}+\left\|\sum_{\alpha=1}^{p} A_{\alpha} \frac{\partial z_{\alpha}}{\partial t}\right\|^{2}=0 .
$$

С помощью интегрирования по частям, с учетом граничных условий и вида оператоpa $A_{\alpha}, \alpha=\overline{1, p}$, из $(2.4)$ находим

$$
\frac{1}{2} \frac{\partial}{\partial t} \sum_{\alpha=1}^{p}\left(k_{\alpha}, z_{\alpha x_{\alpha} t}^{2}\right)+\frac{1}{2} \frac{\partial}{\partial t}\left\|\sum_{\alpha=1}^{p} A_{\alpha} z_{\alpha}\right\|^{2}+\left\|\sum_{\alpha=1}^{p} A_{\alpha} \frac{\partial z_{\alpha}}{\partial t}\right\|^{2}=0 .
$$


Проинтегрируем (2.5) по $\tau$ от 0 до $t$ с учетом условий (2.3). Тогда получим

$$
\frac{1}{2} \sum_{\alpha=1}^{p}\left(k_{\alpha}, z_{\alpha x_{\alpha} t}^{2}\right)+\frac{1}{2}\left\|\sum_{\alpha=1}^{p} A_{\alpha} z_{\alpha}\right\|^{2}+\int_{0}^{t}\left\|\sum_{\alpha=1}^{p} A_{\alpha} \frac{\partial z_{\alpha}}{\partial t}\right\|^{2} d \tau=0 .
$$

Откуда с помощью (2.2) получаем

$$
\frac{\partial^{2} z_{\alpha}}{\partial t^{2}}=0, \quad z_{\alpha}(0)=z_{\alpha t}(0)=0, \quad \alpha=\overline{1, p}
$$

Из (2.6) находим, что $z_{\alpha}(t)=0$ или $u_{\alpha}(t)=u(t), \alpha=\overline{1, p}$.

Для получения априорной оценки (2.1) умножим уравнение (1.5) скалярно на $A_{\alpha} \frac{\partial u_{\alpha}}{\partial t}$ и просуммируем полученное равенство по $\alpha, \alpha=\overline{1, p}$ :

$$
\sum_{\alpha=1}^{p}\left(\frac{\partial^{2} u_{\alpha}}{\partial t^{2}}, A_{\alpha} \frac{\partial u_{\alpha}}{\partial t}\right)+\frac{1}{2} \frac{\partial}{\partial t}\left\|\sum_{\alpha=1}^{p} A_{\alpha} u_{\alpha}\right\|^{2}+\left\|\sum_{\alpha=1}^{p} A_{\alpha} u_{\alpha t}\right\|^{2}=\left(f, \sum_{\alpha=1}^{p} A_{\alpha} \frac{\partial u_{\alpha}}{\partial t}\right) .
$$

Так как

$$
\begin{aligned}
& \sum_{\alpha=1}^{p}\left(\frac{\partial^{2} u_{\alpha}}{\partial t^{2}}, A_{\alpha} \frac{\partial u_{\alpha}}{\partial t}\right)=\frac{1}{2} \frac{\partial}{\partial t} \sum_{\alpha=1}^{p}\left(k_{\alpha}, u_{\alpha x_{\alpha} t}^{2}\right) \\
& \left(f, \sum_{\alpha=1}^{p} A_{\alpha} \frac{\partial u_{\alpha}}{\partial t}\right) \leqslant \frac{1}{2}\|f\|^{2}+\frac{1}{2}\left\|\sum_{\alpha=1}^{p} A_{\alpha} \frac{\partial u_{\alpha}}{\partial t}\right\|^{2}
\end{aligned}
$$

то из $(2.7)$ имеем

$$
c_{1} \sum_{\alpha=1}^{p}\left\|u_{\alpha x_{\alpha} t}\right\|^{2}+\left\|\sum_{\alpha=1}^{p} A_{\alpha} u_{\alpha}\right\|^{2} \leqslant\|f\|_{2, Q_{t}}^{2}+\left\|A u_{0}\right\|^{2}+c_{2} \sum_{\alpha=1}^{p}\left\|u_{1 x_{\alpha}}\right\|^{2},
$$

откуда следует неравенство (2.1). $\triangleright$

\section{3. Устойчивость разностной схемы}

Для приближенного решения задачи (1.5)-(1.6) будем использовать трехслойную векторную аддитивную схему

$$
\begin{gathered}
y_{\alpha \bar{t} t}+\sum_{\beta=1}^{\alpha} A_{h \beta} \hat{y}_{\beta}+\sum_{\beta=\alpha+1}^{p} A_{h \beta} y_{\beta}+\sum_{\beta=1}^{\alpha} A_{h \beta} y_{\beta t}+\sum_{\beta=\alpha+1}^{p} A_{h \beta} \check{y}_{\beta t}=f, \\
y_{\alpha}(0)=u_{0}, \quad y_{\alpha t}(0)=u_{1}, \quad \alpha=1,2, \ldots, p
\end{gathered}
$$

где

$$
\begin{gathered}
A_{h \alpha} y_{i_{\alpha}}=-\left(a_{\alpha} y_{\bar{x}_{\alpha}}\right)_{x_{\alpha}, i_{\alpha}}=-\frac{1}{h_{\alpha}}\left(a_{\alpha}^{\left(i_{\alpha}+1\right)} \frac{y_{i_{\alpha}+1}-y_{i_{\alpha}}}{h_{\alpha}}-a_{\alpha}^{\left(i_{\alpha}\right)} \frac{y_{i_{\alpha}}-y_{i_{\alpha}-1}}{h_{\alpha}}\right), \\
\alpha^{\left(i_{\alpha}\right)}=k_{\alpha}\left(x_{1}, \ldots, x_{\alpha}^{i_{\alpha}-1 / 2}, \ldots, x_{p}\right), \quad y_{t}=(\hat{y}-y) / \tau, \quad y_{\alpha \bar{t} t}=\left(\hat{y}_{i_{\alpha}}-2 y_{i_{\alpha}}+\check{y}_{i_{\alpha}}\right) / \tau^{2}, \quad \alpha=\overline{1, p}
\end{gathered}
$$

- разностные операторы, аппроксимирующие на сетке

$$
\begin{gathered}
\omega_{h \tau}=\bar{\omega}_{h} \times \omega_{\tau}, \quad \bar{\omega}_{h}=\prod_{\alpha=1}^{p} \bar{\omega}_{h_{\alpha}}, \\
\bar{\omega}_{h}=\left\{x_{\alpha}^{\left(i_{\alpha}\right)}=i_{\alpha} h_{\alpha}: \alpha=\overline{1, p} ; i_{\alpha}=0,1, \ldots, N_{\alpha}, h_{\alpha}=l_{\alpha} / N_{\alpha}\right\}, \\
\omega_{\tau}=\left\{t_{j}=j \tau: j=0,1, \ldots, j_{0}\right\}
\end{gathered}
$$

дифференциальные операторы $A_{\alpha} u=-\frac{\partial}{\partial x_{\alpha}}\left(k_{\alpha}(x) \frac{\partial u}{\partial x_{\alpha}}\right), \frac{\partial^{2} u}{\partial t^{2}}, \hat{y}=y^{j+1}, y=y^{j}, \check{y}=y^{j-1}$. 
Введем, как обычно, скалярные произведения и нормы

$$
\begin{gathered}
(y, v)=\sum_{i_{1}=1}^{N_{1}-1} \sum_{i_{2}=1}^{N_{2}-1} \ldots \sum_{i_{\alpha}=1}^{N_{\alpha}-1} \ldots \sum_{i_{p}=1}^{N_{p}-1} y_{i_{1} i_{2} \ldots i_{\alpha} \ldots i_{p}} v_{i_{1} i_{2} \ldots i_{\alpha} \ldots i_{p}} h_{1} h_{2} \ldots h_{\alpha} \ldots h_{p}=\sum_{\omega_{h}} y(x) v(x) H, \\
H=\prod_{\alpha=1}^{p} h_{\alpha}, \quad\|y\|^{2}=(y, y), \\
(y, v]_{\alpha}=\sum_{i_{1}=1}^{N_{1}-1} \sum_{i_{2}=1}^{N_{2}-1} \ldots \sum_{i_{\alpha}=1}^{N_{\alpha}} \ldots \sum_{i_{p}=1}^{N_{p}-1} y_{i_{1} i_{2} \ldots i_{\alpha} \ldots i_{p}} v_{i_{1} i_{2} \ldots i_{\alpha} \ldots i_{p}} H, \quad\|y\|_{\alpha}^{2}=(y, y]_{\alpha} .
\end{gathered}
$$

Из контекста будет ясно о какой норме идет речь, поэтому, чтобы не загромождать изложение, индекс $\alpha$ при написании нормы будем опускать, например, $\left\|y_{\alpha \bar{x}_{\alpha} t}\right\| \equiv\left\|y_{\alpha \bar{x}_{\alpha} t}\right\|_{\alpha}$.

Вычитая из уравнения (3.1) уравнение для нахождения $\hat{y}_{\alpha-1}$, получим

$$
y_{\alpha \bar{t} t}+\tau A_{\alpha} y_{\alpha t}+\tau A_{\alpha} y_{\alpha \bar{t} t}=y_{\alpha-1, \bar{t} t}, \quad \alpha=\overline{2, p} .
$$

Здесь и далее в обозначении $A_{h \alpha}$ будем опускать $h$. Рассматривая уравнение для определения $\hat{y}_{1}$ и $y_{p}$, из (3.1) находим

$$
y_{1 \bar{t} t}+\tau A_{1} y_{1 t}+\tau A_{1} y_{1 \bar{t} t}=\tau \check{f}_{t}+\check{y}_{p \bar{t} t} .
$$

Умножим уравнение (3.3) скалярно на $y_{\alpha \bar{t} t}$ :

$$
\left\|y_{\alpha \bar{t} t}\right\|^{2}+\tau\left(A_{\alpha} y_{\alpha t}, y_{\alpha \bar{t} t}\right)+\tau\left(A_{\alpha} y_{\alpha \bar{t} t}, y_{\alpha \bar{t} t}\right)=\left(y_{\alpha-1, \bar{t} t}, y_{\alpha \bar{t} t}\right) .
$$

Так как

$$
\left(A_{\alpha} y_{\alpha t}, y_{\alpha \bar{t} t}\right)=\left(a_{\alpha} y_{\alpha \bar{x}_{\alpha} t}, y_{\alpha \bar{x}_{\alpha} \bar{t} t}\right]=\frac{1}{2}\left(a_{\alpha}, y_{\alpha \bar{x}_{\alpha} t}^{2}\right]_{\bar{t}}+\frac{\tau}{2}\left(a_{\alpha}, y_{\alpha \bar{x}_{\alpha} \bar{t} t}^{2}\right]
$$

то из (3.5) в силу положительности операторов $A_{\alpha}, \alpha=\overline{1, p}$, имеем

$$
\left\|y_{\alpha \bar{t} t}\right\|^{2}+\left(a_{\alpha}, y_{\alpha \bar{x}_{\alpha} t}^{2}\right] \leqslant\left(a_{\alpha}, \check{y}_{\alpha \bar{x}_{\alpha} t}^{2}\right]+\left\|y_{\alpha-1, \bar{t} t}\right\|^{2},
$$

$a_{\alpha}=k_{\alpha}\left(x_{1}, \ldots, x_{\alpha-1}, x_{\alpha}-0.5 h_{\alpha}, x_{\alpha+1}, \ldots, x_{p}\right)$.

После умножения на $\tau$ просуммируем сначала (3.6) по $j^{\prime}$ от 2 до $j$ :

$$
\sum_{j^{\prime}=2}^{j}\left\|y_{\alpha \bar{t} t}^{j^{\prime}}\right\|^{2} \tau \leqslant c_{2}\left\|y_{\alpha \bar{x} \alpha t}^{1}\right\|^{2} \tau+\sum_{j^{\prime}=2}^{j}\left\|y_{\alpha-1, \bar{t} t}^{j^{\prime}}\right\|^{2} \tau,
$$

а затем по $\alpha^{\prime}$ от 2 до $\alpha$ :

$$
\sum_{j^{\prime}=2}^{j}\left\|y_{\alpha \bar{t} t}^{j^{\prime}}\right\|^{2} \tau \leqslant c_{2} \sum_{\alpha^{\prime}=2}^{\alpha}\left\|y_{\alpha^{\prime} \bar{x}_{\alpha} t}^{1}\right\|^{2} \tau+\sum_{j^{\prime}=2}^{j}\left\|y_{1, \bar{t} t}^{j^{\prime}}\right\|^{2} \tau .
$$

Умножив равенство (3.4) скалярно на $y_{1 \bar{t} t}$, получим

$$
0.5\left\|y_{1 \bar{t} t}\right\|^{2}+\tau\left(A_{1} y_{1 t}, y_{1 \bar{t} t}\right) \leqslant 0.5\left\|\tau \check{f}_{t}+\check{y}_{p \bar{t}}\right\|^{2} .
$$


Пользуясь формулой суммирования по частям и определением оператора $A_{1}$, второе слагаемое в (3.8) запишем в виде

$$
\tau\left(A_{1} y_{1 t}, y_{1 \bar{t} t}\right)=-\tau\left(\left(a_{1} y_{1 \bar{x}_{1} t}\right)_{x_{1}}, y_{1 \bar{t} t}\right)=\tau\left(a_{1} y_{1 \bar{x}_{1} t}, y_{1 \bar{x}_{1} \bar{t} t}\right]=\frac{\tau}{2}\left(a_{1}, y_{1 \bar{x}_{1} t}^{2}\right]_{\bar{t}}+\frac{\tau^{2}}{2}\left(a_{1}, y_{1 \bar{x}_{1} \bar{t} t}^{2}\right]
$$

Умножим (3.8) на $\tau$ и просуммируем полученное неравенство по $j^{\prime}$ от 2 до $j$ :

$$
\sum_{j^{\prime}=2}^{j}\left\|y_{1 \bar{t} t}^{j^{\prime}}\right\|^{2} \tau+\left(a_{1},\left(y_{1 \bar{x}_{1} t}^{j}\right)^{2}\right] \tau \leqslant\left(a_{1},\left(y_{1 \bar{x}_{1} t}^{1}\right)^{2}\right] \tau+\tau \sum_{j^{\prime}=2}^{j}\left\|\tau \check{f}_{t}+\check{y}_{p \overline{t t}}\right\|^{2}
$$

или

$$
\sum_{j^{\prime}=2}^{j}\left\|y_{1 \bar{t} t}^{j^{\prime}}\right\|^{2} \tau \leqslant c_{2}\left\|y_{1 \bar{x}_{1} t}^{1}\right\|^{2}+\tau \sum_{j^{\prime}=2}^{j}\left\|\tau \check{f}_{t}+\check{y}_{p \bar{t} t}\right\|^{2}, \quad 0<c_{1} \leqslant a_{2} \leqslant c_{2} .
$$

Так как

$$
\begin{aligned}
& \left\|\tau \check{f}_{t}+y_{p \bar{t} t}\right\|^{2} \leqslant\left(\tau\left\|\check{f}_{t}\right\|+\left\|y_{p \bar{t} t}\right\|\right)^{2}=\tau^{2}\left\|\check{f}_{t}\right\|^{2}+2 \sqrt{\bar{\tau}}\left\|\check{f}_{t}\right\| \cdot \sqrt{\bar{\tau}}\left\|\check{y}_{p \bar{t} t}\right\|+\left\|\check{y}_{p \bar{t} t}\right\|^{2} \\
& \leqslant \tau^{2}\left\|\check{f}_{t}\right\|^{2}+\tau\left\|\check{f}_{t}\right\|^{2}+\tau\left\|\check{y}_{p \bar{t} t}\right\|^{2}+\left\|\check{y}_{p \bar{t} t}\right\|^{2}=(1+\tau) \tau\left\|\check{f}_{t}\right\|^{2}+(1+\tau)\left\|\check{y}_{p \bar{t}}\right\|^{2}
\end{aligned}
$$

то из (3.8) после суммирования по $j^{\prime}$ от 2 до $j$ получаем

$$
\begin{gathered}
\sum_{j^{\prime}=2}^{j}\left\|y_{1 \bar{t} t}^{j^{\prime}}\right\|^{2} \tau \leqslant c_{2}\left\|y_{1 \bar{x}_{1} t}^{1}\right\|^{2} \tau+(1+\tau) \tau \sum_{j^{\prime}=2}^{j}\left\|f_{t}^{j^{\prime}-1}\right\|^{2} \tau \\
+(1+\tau) \tau\left\|y_{p \bar{t} t}^{1}\right\|^{2}+(1+\tau) \sum_{j^{\prime}=2}^{j-1}\left\|y_{p \bar{t} t}^{j^{\prime}}\right\|^{2} \tau
\end{gathered}
$$

Оценим последнее слагаемое (3.9) с помощью неравенства (3.7):

$$
\sum_{j^{\prime}=2}^{j}\left\|y_{p \bar{t} t}^{j^{\prime}}\right\|^{2} \tau \leqslant c_{2} \sum_{\alpha=2}^{p}\left\|y_{\alpha \bar{x}_{\alpha} t}^{1}\right\|^{2} \tau+\sum_{j^{\prime}=2}^{j-1}\left\|y_{1 \bar{t} t}^{j^{\prime}}\right\|^{2} \tau
$$

Из (3.9) с помощью (3.10) находим

$$
\begin{gathered}
\sum_{j^{\prime}=2}^{j}\left\|y_{1 \bar{t} t}^{j^{\prime}}\right\|^{2} \tau \leqslant(1+\tau) c_{2} \sum_{\alpha=1}^{p}\left\|y_{\alpha \bar{x}_{\alpha} t}^{1}\right\|^{2} \tau \\
+(1+\tau) \tau \sum_{j^{\prime}=2}^{j}\left\|f_{t}^{j^{\prime}-1}\right\|^{2} \tau+(1+\tau) \tau\left\|y_{p \bar{t} t}^{1}\right\|^{2}+(1+\tau) \sum_{j^{\prime}=2}^{j-1}\left\|y_{1 \bar{t} t}^{j^{\prime}}\right\|^{2} \tau .
\end{gathered}
$$

Продолжая неравенство (3.11) вправо с помощью аналогичной процедуры, найдем

$$
\sum_{j^{\prime}=2}^{j}\left\|y_{1 \bar{t} t}^{j^{\prime}}\right\|^{2} \tau \leqslant M_{1}(T)\left(\left\|y_{1 \bar{t} t}^{1}\right\|^{2}+\left\|y_{p \bar{t} t}^{1}\right\|^{2}+\sum_{j^{\prime}=2}^{j}\left\|f_{t}^{j^{\prime}-1}\right\|^{2} \tau+\sum_{\alpha=1}^{p}\left\|y_{\alpha \bar{x}_{\alpha} t}^{1}\right\|^{2}\right)
$$

где $M_{1}(T)=c_{3} T e^{T}, c_{3}=\max \left(1, c_{2}\right)$. 
Из (3.7) и (3.12) следует

$$
\sum_{j^{\prime}=2}^{j}\left\|y_{\alpha \bar{t} t}^{j^{\prime}}\right\|^{2} \tau \leqslant M_{2}(T)\left(\left\|y_{1 \bar{t} t}^{1}\right\|^{2}+\left\|y_{p \bar{t} t}^{1}\right\|^{2}+\sum_{j^{\prime}=2}^{j}\left\|f_{t}^{j^{\prime}-1}\right\|^{2} \tau+\sum_{\alpha=1}^{p}\left\|y_{\alpha \bar{x}_{\alpha}}^{1}\right\|^{2}\right) .
$$

Так как из (3.6) при $j=1$ следует

$$
\left\|y_{p \bar{t} t}^{1}\right\|^{2} \leqslant c_{2} \sum_{\alpha=2}^{p}\left\|y_{\alpha \bar{x}_{\alpha} t}^{0}\right\|^{2}+\left\|y_{1 \bar{t} t}^{1}\right\|^{2}
$$

то для получения необходимой оценки достаточно оценить $\left\|y_{1 \bar{t} t}^{1}\right\|$ и $\left\|y_{\alpha \bar{x}_{\alpha}}^{1}\right\|, \alpha=\overline{1, p}$.

Для чего положим в уравнении (3.1) $\alpha=1, j=1$. Тогда получим

$$
y_{1 \bar{t} t}(\tau)+\tau A_{1}\left(y_{1 t}(\tau)+y_{1 \bar{t} t}(\tau)\right)+A u_{1}+A\left(u_{0}+\tau u_{1}\right)=f(\tau) .
$$

Умножая (3.14) скалярно на $y_{1 \bar{t} t}(\tau)$, находим

$$
\left\|y_{1 \bar{t} t}^{1}\right\|^{2} \leqslant c_{2}\left\|u_{1 \bar{x}_{1}} \rrbracket^{2}+\right\| f(\tau)-A \tilde{u}_{1} \|^{2}, \quad \tilde{u}_{1}=u_{0}+(1+\tau) u_{1} .
$$

Из (3.6) с учетом (3.15) имеем

$$
\sum_{\alpha=2}^{p}\left\|y_{\alpha \bar{x}_{\alpha} t}^{1}\right\|^{2} \leqslant \frac{c_{2}}{c_{1}} \sum_{\alpha=2}^{p}\left\|y_{\alpha \bar{x}_{\alpha} t}^{0}\right\|^{2}+\frac{1}{c_{1}}\left(c_{2}\left\|u_{1 \bar{x}_{1}}\right\|^{2}+\left\|f(\tau)-A \tilde{u_{1}}\right\|^{2}\right) .
$$

Аналогично с помощью (3.14) оценивается и $\left\|y_{1 \bar{x}_{1} t}^{1}\right\|^{2}$. С помощью (3.15), (3.16) из (3.13) получаем

$$
\sum_{j^{\prime}=2}^{j}\left\|y_{\alpha \bar{t} t}^{j^{\prime}}\right\|^{2} \tau \leqslant M(T)\left(\sum_{j^{\prime}=2}^{j}\left\|f_{t}^{j^{\prime}-1}\right\|^{2} \tau+\sum_{\alpha=1}^{p}\left\|u_{1 \overline{x_{\alpha}}}\right\|^{2}+\left\|f(\tau)-A \tilde{u}_{1}\right\|^{2}\right), \quad \alpha=\overline{1, p} .
$$

Из оценки (3.17) следует устойчивость разностной схемы (3.1)-(3.2) по правой части и начальным данным.

3.1. Погрешность аппроксимации. Обозначим через $z_{\alpha}=y_{\alpha}-u_{\alpha}, \alpha=\overline{1, p}$, и подставим $y_{\alpha}=z_{\alpha}+u_{\alpha}$ в (3.1)-(3.2). Тогда для погрешности получим задачу

$$
\begin{gathered}
z_{\alpha \bar{t} t}+\sum_{\beta=1}^{\alpha} A_{h \beta} \hat{z}_{\beta}+\sum_{\beta=\alpha+1}^{p} A_{h \beta} z_{\beta}+\sum_{\beta=1}^{\alpha} A_{h \beta} z_{\beta t}+\sum_{\beta=\alpha+1}^{p} A_{h \beta} \check{z}_{\beta t}=\psi_{\alpha}, \\
z_{\alpha}(0)=0, \quad z_{\alpha t}(0)=\nu_{1}, \quad \alpha=1,2, \ldots, p,
\end{gathered}
$$

где

$$
\begin{aligned}
\psi_{\alpha} & =-\left(u_{\alpha \bar{t} t}+\sum_{\beta=1}^{\alpha} A_{h \beta} \hat{u}_{\beta}+\sum_{\beta=\alpha+1}^{p} A_{h \beta} u_{\beta}+\sum_{\beta=1}^{\alpha} A_{h \beta} u_{\beta t}+\sum_{\beta=\alpha+1}^{p} A_{h \beta} \check{u}_{\beta t}\right)+f \\
& =-\left(\frac{d^{2} u}{d t^{2}}+\sum_{\beta=1}^{\alpha} A_{h \beta} u_{\beta}+\sum_{\beta=1}^{\alpha} A_{h \beta} \frac{d u_{\beta}}{d t}\right)+O\left(|h|^{2}+\tau\right)+f=O\left(|h|^{2}+\tau\right),
\end{aligned}
$$


$\nu_{1}=\frac{d^{2} u}{d t^{2}}(\xi) \frac{\tau}{2}=O(\tau), 0<\xi<\tau,|h|=h_{1}^{2}+h_{2}^{2}+\ldots+h_{p}^{2}, O\left(|h|^{2}\right)-$ погрешность аппроксимации оператора $A$. Кроме того, если $u_{\alpha} \in C^{4}[0, T], f(t) \in C^{2}[0, T]$, то

$$
\begin{gathered}
\psi_{\alpha \bar{t}}=-\left(\frac{d^{3} u}{d t^{3}}+\sum_{\beta=1}^{\alpha} A_{h \beta} \frac{d u_{\beta}}{d t}+\sum_{\beta=1}^{\alpha} A_{h \beta} \frac{d^{2} u_{\beta}}{d t^{2}}\right)+O\left(|h|^{2}+\tau\right)+f_{\bar{t}} \\
=-\frac{d f}{d t}+f_{\bar{t}}+O\left(|h|^{2}+\tau\right)=O\left(|h|^{2}+\tau\right),
\end{gathered}
$$

$C^{n}[0, T]$ - класс функций, имеющий $n$ производных, непрерывных по $t$ на $[0, T]$.

3.2. Сходимость разностной схемы. Для погрешности $z_{\alpha}=y_{\alpha}-u_{\alpha}$ оценка (3.17) принимает вид

$$
\sum_{j^{\prime}=2}^{j}\left\|z_{\alpha \bar{t} t}^{j^{\prime}}\right\|^{2} \tau \leqslant M\left(\sum_{j^{\prime}=2}^{j}\left\|\psi_{\alpha \bar{t}}^{j^{\prime}}\right\|^{2} \tau+\sum_{\alpha=1}^{p}\left\|\nu_{1 \bar{x}_{\alpha}}\right\|^{2}+\left\|\psi_{\alpha}(\tau)+(1+\tau) A \nu_{1}\right\|^{2}\right) .
$$

Из оценки (3.21) с учетом (3.20) следует

Теорема. Пусть задача (1.1)-(1.2) имеет единственное непрерывное в $\bar{Q}_{T}$ решение $u(x, t)$ и существуют производные $\frac{\partial^{4} u}{\partial x_{\alpha}^{4}}, \frac{\partial^{4} u}{\partial t^{4}}, \frac{\partial^{8} u}{\partial x_{\alpha}^{4} \partial t^{4}}, \frac{\partial^{2} f}{\partial t^{2}}, \frac{\partial^{2} f}{\partial x_{\alpha}^{2}}, \alpha=\overline{1, p}$, непрерывные в $\bar{Q}_{T}$. Тогда схема (1.1)-(1.2) сходится со скоростью $O\left(|h|^{2}+\tau\right)$ в норме $\|z\|_{1}=\left(\sum_{j^{\prime}=2}^{j}\left\|z_{\alpha \bar{t} t}^{j^{\prime}}\right\|^{2} \tau\right)^{1 / 2}$.

\section{4. Волновое уравнение в релаксирующих средах}

В цилиндре $Q_{T}$ рассмотрим задачу для волнового уравнения в релаксирующих средах

$$
\begin{gathered}
\frac{\partial^{2} u}{\partial t^{2}}+L u=f(x, t), \quad L u=\sum_{\alpha=1}^{p} L_{\alpha} u, \quad(x, t) \in Q_{T}, \\
\left.u\right|_{S}=0, \quad u(x, 0)=u_{0}(x), \quad u_{t}(x, 0)=u_{1}(x), \quad \chi_{1}, \chi_{2}=\text { const }>0,
\end{gathered}
$$

где $L_{\alpha} u=-\left[\frac{\partial^{2} u}{\partial x_{\alpha}^{2}}+\chi_{1} \frac{\partial}{\partial t} \frac{\partial^{2} u}{\partial x_{\alpha}^{2}}+\chi_{2} \frac{\partial^{2}}{\partial t^{2}} \frac{\partial^{2} u}{\partial x_{\alpha}^{2}}\right]$.

Структура третьего члена слева уравнения (4.1) такая же как у диссипативного члена в (1.1), описывающего затухания звука из-за вязкости и теплопроводности, четвертый член описывает слабые дисперсионные эффекты (см. [13, с. 87]).

Задачу (4.1)-(4.2) перепишем в виде абстрактной задачи Коши

$$
\begin{gathered}
\frac{d^{2} u}{d t^{2}}+A u+\chi_{1} A \frac{d u}{d t}+\chi_{2} A \frac{d^{2} u}{d t^{2}}=f(t), \quad A=\sum_{\alpha=1}^{p} A_{\alpha}, \quad A_{\alpha}=-\frac{\partial^{2}}{\partial x_{\alpha}^{2}}, \\
u(0)=u_{0}, \quad u_{t}(0)=u_{1} .
\end{gathered}
$$

Вместо исходной задачи (4.3)-(4.4) запишем систему подзадач

$$
\begin{gathered}
\frac{d^{2} u_{\alpha}}{d t^{2}}+\sum_{\beta=1}^{p} A_{\beta} u_{\beta}+\chi_{1} \sum_{\beta=1}^{p} A_{\beta} u_{\beta t}+\chi_{2} \sum_{\beta=1}^{p} A_{\beta} u_{\beta t t}=f, \quad \alpha=\overline{1, p} \\
u_{\alpha}(0)=u_{0}, \quad u_{\alpha t}(0)=u_{1} .
\end{gathered}
$$


Как и выше, обозначим через $z_{\alpha}=u_{\alpha}-u$. Подставляя $u_{\alpha}=z_{\alpha}+u$ в уравнение (4.5), получим

$$
\begin{gathered}
\frac{\partial^{2} z_{\alpha}}{\partial t^{2}}+\sum_{\beta=1}^{p} A_{\beta} z_{\beta}+\chi_{1} \sum_{\beta=1}^{p} A_{\beta} z_{\beta t}+\chi_{2} \sum_{\beta=1}^{p} A_{\beta} z_{\beta t t}=0, \\
z_{\alpha}(0)=z_{\alpha t}(0)=0, \quad \alpha=\overline{1, p} .
\end{gathered}
$$

Умножив каждое из уравнений (4.7) скалярно на $A_{\alpha} \frac{\partial z_{\alpha}}{\partial t}$ и просуммировав их по $\alpha=$ $\overline{1, p}$, получим

$$
\begin{aligned}
\sum_{\alpha=1}^{p}\left(\frac{\partial^{2} z_{\alpha}}{\partial t^{2}}, A_{\alpha} \frac{\partial z_{\alpha}}{\partial t}\right) & +\sum_{\alpha=1}^{p}\left(\sum_{\beta=1}^{p} A_{\beta} z_{\beta}, A_{\alpha} \frac{\partial z_{\alpha}}{\partial t}\right)+\chi_{1} \sum_{\alpha=1}^{p}\left(\sum_{\beta=1}^{p} A_{\beta} z_{\beta t}, A_{\alpha} \frac{\partial z_{\alpha}}{\partial t}\right) \\
& +\chi_{2} \sum_{\alpha=1}^{p}\left(\sum_{\beta=1}^{p} A_{\beta} z_{\beta t t}, A_{\alpha} \frac{\partial z_{\alpha}}{\partial t}\right)=0 .
\end{aligned}
$$

После интегрирования по частям в первом интеграле равенства (4.9) находим

$$
\sum_{\alpha=1}^{p}\left\|z_{\alpha x_{\alpha} t}\right\|^{2}+\left\|\sum_{\alpha=1}^{p} A_{\alpha} z_{\alpha}\right\|^{2}+2 \chi_{1} \int_{0}^{t}\left\|A_{\alpha} z_{\alpha t}\right\|^{2} d \tau+\chi_{2}\left\|\sum_{\alpha=1}^{p} A_{\alpha} z_{\alpha t}\right\|^{2}=0 .
$$

Откуда следует, что $\sum_{\alpha=1}^{p} A_{\alpha} z_{\alpha}=\sum_{\alpha=1}^{p} A_{\alpha} z_{\alpha t}=\sum_{\alpha=1}^{p} A_{\alpha} z_{\alpha t t}=0$, т. е. задача (4.7)-(4.8) принимает вид

$$
\frac{\partial^{2} z_{\alpha}}{\partial t^{2}}=0, \quad z_{\alpha}(0)=z_{\alpha t}(0)=0 .
$$

Из (4.10) имеем, что для любого $t \in[0, T], z_{\alpha}(t)=0$ или, что тоже самое, $u_{\alpha}(t)=u(t)$, $t \in[0, T], \alpha=\overline{1, p}$.

Покажем, что каждая из компонент вектор-решения непрерывно зависит от входных данных. Умножим уравнение (4.5) скалярно на $A_{\alpha} u_{\alpha t}$, затем просуммируем их по $\alpha=$ $\overline{1, p}$. В результате получим

$$
\begin{gathered}
\frac{1}{2} \frac{\partial}{\partial t} \sum_{\alpha=1}^{p}\left\|u_{\alpha x_{\alpha} t}\right\|^{2}+\frac{1}{2} \frac{\partial}{\partial t}\left\|\sum_{\alpha=1}^{p} A_{\alpha} u_{\alpha}\right\|^{2}+\chi_{1}\left\|\sum_{\alpha=1}^{p} A_{\alpha} u_{\alpha t}\right\|^{2} \\
+\frac{\chi_{2}}{2} \frac{\partial}{\partial t}\left\|\sum_{\alpha=1}^{p} A_{\alpha} u_{\alpha t}\right\|^{2} \leqslant \frac{1}{4 \varepsilon}\|f\|^{2}+\varepsilon\left\|\sum_{\alpha=1}^{p} A_{\alpha} u_{\alpha t}\right\|^{2} .
\end{gathered}
$$

Положив $\varepsilon=\frac{\chi_{1}}{2}$, затем проинтегрировав (4.11) по $\tau$ от 0 до $t$, получим

$$
\begin{gathered}
\sum_{\alpha=1}^{p}\left\|u_{\alpha x_{\alpha} t}\right\|^{2}+\left\|\sum_{\alpha=1}^{p} A_{\alpha} u_{\alpha}\right\|^{2}+\chi_{2}\left\|\sum_{\alpha=1}^{p} A_{\alpha} u_{\alpha t}\right\|^{2} \\
\leqslant \frac{1}{\chi_{1}}\|f\|_{2, Q_{t}}^{2}+\left\|u_{1 x}\right\|^{2}+\left\|A u_{0}\right\|^{2}+\left\|A u_{1}\right\|^{2}, \\
\|f\|_{2, Q_{t}}^{2}=\int_{0}^{t}\|f\|^{2} d \tau .
\end{gathered}
$$

Из оценки (4.12) следует непрерывная зависимость решения задачи (4.5)-(4.6) от правой части и начальных данных. 


\section{5. Разностные схемы}

Для решения задачи (4.1)-(4.2) будем использовать векторную аддитивную схему

$$
\begin{gathered}
y_{\alpha \bar{t} t}+\sum_{\beta=1}^{\alpha} A_{\beta} \hat{y}_{\beta}+\sum_{\beta=\alpha+1}^{p} A_{\beta} y_{\beta}+\chi_{1} \sum_{\beta=1}^{\alpha} A_{\beta} y_{\beta t}+\chi_{1} \sum_{\beta=\alpha+1}^{p} A_{\beta} \check{y}_{\beta t} \\
+\chi_{2} \sum_{\beta=1}^{\alpha} A_{\beta} y_{\beta \bar{t} t}+\chi_{2} \sum_{\beta=\alpha+1}^{p} A_{\beta} \check{y}_{\beta \bar{t} t}=f, \\
y_{\alpha}(0)=u_{0}, \quad y_{\alpha t}(0)=u_{1} .
\end{gathered}
$$

Задача (5.1)-(5.2) недоопределена. Для применения этой схемы требуется задать еще одно условие, например, значение $y_{\alpha}(t)$ на втором слое $y_{\alpha}(2 \tau)$.

Будем искать $y_{\alpha}(2 \tau)$ из соотношения

$$
\frac{u(2 \tau)-2 u(\tau)+u(0)}{\tau^{2}}=\frac{\partial^{2} u}{\partial t^{2}}(\tau)+O\left(\tau^{2}\right) .
$$

Определим $\frac{\partial^{2} u}{\partial t^{2}}(\tau)$ из уравнения (4.1), положив $t=\tau$. Тогда получим задачу для определения $v=\frac{\partial^{2} u}{\partial t^{2}}(x, \tau)$

$$
\begin{gathered}
\Delta v-\frac{1}{\chi_{2}} v=F,\left.\quad v\right|_{\Gamma}=0, \\
F=\frac{1}{\chi_{2}}\left[f(x, t)+\sum_{\alpha=1}^{p} \frac{\partial^{2} u}{\partial x_{\alpha}^{2}}(x, \tau)+\sum_{\alpha=1}^{p} \frac{\partial^{2} \dot{u}}{\partial x_{\alpha}^{2}}(x, \tau)\right], \quad \dot{u}=\frac{\partial u}{\partial t} .
\end{gathered}
$$

Так как $\chi_{2}>0$, то задача Дирихле (5.3) однозначно разрешима.

Итак, будем считать, что задача (5.1)-(5.2) дополнена еще одним условием

$$
y_{\alpha \bar{t} t}=u_{2} \quad \text { или } \quad y_{\alpha}(2 \tau)=\bar{u}_{2}, \quad \bar{u}_{2}=u_{0}+2 u_{1} \tau+u_{2} \tau^{2} .
$$

Вычитая из уравнения (5.1) уравнение для определения $\hat{y}_{\alpha-1}$, получим

$$
y_{\alpha \bar{t} t}+\tau A_{\alpha} y_{\alpha t}+\chi_{1} \tau A_{\alpha} y_{\alpha \bar{t} t}+\chi_{2} \tau A_{\alpha} y_{\alpha \bar{t} \bar{t} t}=y_{\alpha-1 . \bar{t} t} .
$$

Умножим (5.4) скалярно на $y_{\alpha \bar{t} t}$ :

$$
\left\|y_{\alpha \bar{t} t}\right\|^{2}+\tau\left(A_{\alpha} y_{\alpha t}, y_{\alpha \bar{t} t}\right)+\chi_{1} \tau\left(A_{\alpha} y_{\alpha \bar{t} t}, y_{\alpha \bar{t} t}\right)+\chi_{2} \tau\left(A_{\alpha} y_{\alpha \bar{t} \bar{t} t}, y_{\alpha \bar{t} t}\right)=\left(y_{\alpha-1 . \bar{t} t}, y_{\alpha \bar{t} t}\right) .
$$

Откуда с учетом вида операторов $A_{\alpha}$ находим

$$
\left\|y_{\alpha \bar{t} t}\right\|^{2}+\left\|y_{\alpha \bar{x}_{\alpha} t}\right\|^{2}+\chi_{2}\left\|y_{\alpha \bar{x}_{\alpha} \bar{t} t}\right\|^{2} \leqslant\left\|\check{y}_{\alpha \bar{x}_{\alpha} t}\right\|^{2}+\chi_{2}\left\|\check{y}_{\alpha \bar{x}_{\alpha} \bar{t} t}\right\|^{2}+\left\|y_{\alpha-1, \bar{t} t}\right\|^{2} .
$$

Просуммировав (5.5) сначала по $j^{\prime}$ от 3 до $j$, а затем по $s$ от 2 до $\alpha$, получим

$$
\sum_{j^{\prime}=3}^{j}\left\|y_{\alpha \bar{t} t}^{j^{\prime}}\right\|^{2} \tau \leqslant \sum_{s=2}^{\alpha}\left\|y_{s \bar{x}_{s} t}(2 \tau)\right\|^{2} \tau+\chi_{2} \sum_{s=2}^{\alpha}\left\|y_{s \bar{x}_{s} \bar{t} t}(2 \tau)\right\|^{2} \tau+\sum_{j^{\prime}=3}^{j}\left\|y_{1 \bar{t} t}^{j^{\prime}}\right\|^{2} \tau .
$$

Рассматривая уравнения для определения $\hat{y}_{1}$ и $y_{p}$ из (5.1), получаем равенство

$$
y_{1 \bar{t} t}+\tau A_{1} y_{1 t}+\chi_{1} \tau A_{1} y_{1 \bar{t} t}+\chi_{2} \tau A_{1} y_{1 \bar{t} \bar{t} t}=\tau \check{f}_{t}+\check{y}_{p \bar{t} t} .
$$


Из (5.7), как и выше, с помощью (5.6) получаем послойную оценку

$$
\begin{aligned}
& \sum_{j^{\prime}=3}^{j}\left\|y_{1 \bar{t} t}^{j^{\prime}}\right\|^{2} \tau \leqslant(1+\tau) \sum_{\alpha=1}^{p}\left\|y_{\alpha \bar{x}_{\alpha} t}(2 \tau)\right\|^{2} \tau+(1+\tau) \chi_{2} \sum_{\alpha=1}^{p}\left\|y_{\alpha \bar{x}_{\alpha} \bar{t} t}(2 \tau)\right\|^{2} \tau \\
& +(1+\tau) \sum_{j^{\prime}=3}^{j-1}\left\|y_{1 \bar{t} t}^{j^{\prime}}\right\|^{2} \tau+(1+\tau) \tau \sum_{j^{\prime}=3}^{j}\left\|f_{t}^{j^{\prime}-1}\right\|^{2} \tau+(1+\tau) \tau\left\|y_{1 \bar{t} t}(2 \tau)\right\|^{2} .
\end{aligned}
$$

Продолжая неравенство (5.8) вправо, находим

$$
\begin{gathered}
\sum_{j^{\prime}=3}^{j}\left\|y_{1 \bar{t} t}^{j^{\prime}}\right\|^{2} \tau \leqslant M(T)\left(\left\|y_{1 \bar{t} t}(2 \tau)\right\|^{2}+\left\|y_{p \bar{t} t}(2 \tau)\right\|^{2}\right. \\
\left.+\sum_{j^{\prime}=3}^{j}\left\|f_{t}^{j^{\prime}-1}\right\|^{2} \tau+\sum_{\alpha=1}^{p}\left\|y_{\alpha \bar{x}_{\alpha} t}(2 \tau)\right\|^{2}+\chi_{2} \sum_{\alpha=1}^{p}\left\|y_{\alpha \bar{x}_{\alpha} \bar{t} t}(2 \tau)\right\|^{2}\right) .
\end{gathered}
$$

Из (5.6) и (5.9) получаем оценку

$$
\begin{gathered}
\sum_{j^{\prime}=3}^{j}\left\|y_{\alpha \bar{t} t}^{j^{\prime}}\right\|^{2} \tau \leqslant M(T)\left(\left\|y_{1 \bar{t} t}(2 \tau)\right\|^{2}+\left\|y_{p \bar{t} t}(2 \tau)\right\|^{2}+\sum_{j^{\prime}=3}^{j}\left\|f_{t}^{j^{\prime}-1}\right\|^{2} \tau\right. \\
\left.+\sum_{\alpha=1}^{p}\left\|y_{\alpha \bar{x}_{\alpha} t}(2 \tau)\right\|^{2}+\sum_{\alpha=1}^{p}\left\|y_{\alpha \bar{x}_{\alpha} \bar{t} t}(2 \tau)\right\|^{2}\right) .
\end{gathered}
$$

Так как из (5.5) следует

$$
\left\|y_{p \bar{t} t}(2 \tau)\right\|^{2} \leqslant \sum_{\alpha=2}^{p}\left\|y_{\alpha \bar{x}_{\alpha} t}(\tau)\right\|^{2}+\chi_{2} \sum_{\alpha=2}^{p}\left\|y_{\alpha \bar{x}_{\alpha} \bar{t} t}(\tau)\right\|^{2}+\left\|y_{1 \bar{t} t}(2 \tau)\right\|^{2},
$$

то для получения необходимой оценки достаточно оценить $\left\|y_{1 \bar{t} t}(2 \tau)\right\|,\left\|y_{\alpha \bar{x}_{\alpha} t}(2 \tau)\right\|$, $\left\|y_{\alpha \bar{x}_{\alpha} \bar{t} t}(2 \tau)\right\|, \alpha=\overline{1, p}$.

Для этого положим в (5.1) $\alpha=1, j=2$ :

$$
\begin{gathered}
y_{1 \bar{t} t}(2 \tau)+A_{1} y_{1}(3 \tau)+\sum_{\beta=2}^{p} A_{\beta} y_{\beta}(2 \tau)+\chi_{1} A_{1} y_{1 t}(2 \tau) \\
+\chi_{1} \sum_{\beta=2}^{p} A_{\beta} y_{\beta t}(\tau)+\chi_{2} A_{1} y_{1 \bar{t} t}(2 \tau)+\chi_{2} \sum_{\beta=2}^{p} A_{\beta} y_{\beta \overline{t t}}(\tau)=f(2 \tau) .
\end{gathered}
$$

Из (5.11) следует

$$
y_{1 \bar{t} t}(2 \tau)+\tau A_{1} y_{1 t}(2 \tau)+\tau \chi_{1} A_{1} y_{1 \bar{t} t}(2 \tau)+\tau \chi_{2} A_{1} y_{1 \bar{t} t \bar{t}}(2 \tau)=F(2 \tau),
$$

где $F(2 \tau)=f(2 \tau)-A \bar{u}_{2}-\chi_{1} A\left(u_{1}+\tau u_{2}\right)-\chi_{2} A u_{2}$.

Из (5.12) следует

$$
\left\|y_{1 \bar{t} t}(2 \tau)\right\|^{2} \leqslant\left\|y_{1 \bar{x}_{1} t}(\tau)\right\|^{2}+\chi_{2}\left\|y_{1 \bar{x}_{1} \bar{t} t}(\tau)\right\|^{2}+\|F\|^{2} .
$$


Аналогично из (5.5) получаем оценки

$$
\begin{gathered}
\sum_{\alpha=2}^{p}\left\|y_{\alpha \bar{x}_{\alpha} t}(2 \tau)\right\|^{2} \leqslant \sum_{\alpha=2}^{p}\left\|y_{\alpha \bar{x}_{\alpha} t}(\tau)\right\|^{2}+\chi_{2} \sum_{\alpha=2}^{p}\left\|y_{\alpha \bar{x}_{\alpha} \bar{t} t}(\tau)\right\|^{2}+\left\|y_{1 \bar{t} t}(2 \tau)\right\|^{2}, \\
\sum_{\alpha=2}^{p}\left\|y_{\alpha \bar{x}_{\alpha} \bar{t} t}(2 \tau)\right\|^{2} \leqslant \frac{1}{\chi_{2}} \sum_{\alpha=2}^{p}\left\|y_{\alpha \bar{x}_{\alpha} t}(\tau)\right\|^{2}+\sum_{\alpha=2}^{p}\left\|y_{\alpha \bar{x}_{\alpha} \bar{t} t}(\tau)\right\|^{2}+\frac{1}{\chi_{2}}\left\|y_{1 \bar{t} t}(2 \tau)\right\|^{2} .
\end{gathered}
$$

Нормы $\left.\| y_{1 \bar{x}_{1} t}(2 \tau)\right],\left\|y_{1 \bar{x}_{1} \bar{t} t}\right\|$ оцениваются с помощью равенства (5.7). С помощью оценок (5.11), (5.14)-(5.16) из (5.10) окончательно получим

$$
\begin{gathered}
\sum_{j^{\prime}=3}^{j}\left\|y_{\alpha \bar{t} t}^{j^{\prime}}\right\|^{2} \tau \leqslant M\left(\sum_{j^{\prime}=3}^{j}\left\|f_{t}^{j^{\prime}-1}\right\|^{2} \tau+\sum_{\alpha=1}^{p}\left\|u_{1 \bar{x}_{\alpha}}+\bar{u}_{2 \bar{x}_{\alpha}} \tau\right\|^{2}\right. \\
\left.+\sum_{\alpha=1}^{p}\left\|u_{2 \bar{x}_{\alpha}}\right\|^{2}+\left\|f(2 \tau)-A \bar{u}_{2}-\chi_{1} A\left(u_{1}+\tau u_{2}\right)-\chi_{2} A u_{2}\right\|^{2}\right) .
\end{gathered}
$$

Из (5.17) следует устойчивость схемы (5.1)-(5.2) по правой части и начальным условиям, а при достаточной гладкости решения исходной дифференциальной задачи сходимость схемы (5.1)-(5.2) со скоростью $O\left(|h|^{2}+\tau\right)$. Условия гладкости на $u(x, t)$ здесь те же самые, что и в теореме из пункта 3.2 .

\section{6. Модифицированное уравнение влагопереноса}

В цилиндре $Q_{T}$ рассмотрим уравнение влагопереноса Аллера

$$
\begin{gathered}
\frac{\partial u}{\partial t}=L u+f(x, t), \quad L u=\sum_{\alpha=1}^{p} L_{\alpha} u, \quad x \in G, t \in(0, T] \\
\left.u\right|_{S_{T}}=0, \quad u(x, 0)=u_{0}(x),
\end{gathered}
$$

где $L_{\alpha} u=\frac{\partial}{\partial x_{\alpha}}\left(k_{\alpha}(x) \frac{\partial u}{\partial x_{\alpha}}\right)+\frac{\partial}{\partial t} \frac{\partial}{\partial x_{\alpha}}\left(k_{\alpha}(x) \frac{\partial u}{\partial x_{\alpha}}\right), \alpha=\overline{1, p}, 0<c_{1} \leqslant k_{\alpha}(x) \leqslant c_{2}$.

Задачу (6.1)-(6.2) перепишем в виде абстрактной задачи Коши

$$
\begin{gathered}
\frac{d u}{d t}+A u+A \frac{d u}{d t}=f(t), \quad A=\sum_{\alpha=1}^{p} A_{\alpha}, \quad A_{\alpha}=-\frac{\partial}{\partial x_{\alpha}}\left(k_{\alpha}(x) \frac{\partial}{\partial x_{\alpha}}\right), \\
u(0)=u_{0} .
\end{gathered}
$$

Введенный Аллером (см. [8]) дополнительный член $\chi \frac{\partial}{\partial t} \frac{\partial^{2} u}{\partial x^{2}}$ призван объяснить опытный факт движения влаги против градиента влажности. Коэффициент Аллера $\chi$ мал при впитывании влаги и велик при испарении (см. [12, с. 159]). Мы включаем это слагаемое в уравнение в виде $\left(k_{\alpha}(x) u_{x}\right)_{x t}, \alpha=\overline{1, p}$.

Вместо задачи (6.3)-(6.4) запишем систему подзадач:

$$
\begin{gathered}
\frac{d u_{\alpha}}{d t}+\sum_{\beta=1}^{p} A_{\beta} u_{\beta}+\sum_{\beta=1}^{p} A_{\beta} u_{\beta t}=f(t), \\
u_{\alpha}(0)=u_{0}, \quad \alpha=\overline{1, p} .
\end{gathered}
$$


Очевидно, что $u_{\alpha}(t)=u(t)$, и поэтому в качестве решения исходной задачи $(6.5)-(6.6)$ можно взять любую компоненту вектора $U(t)=\left(u_{1}(t), \ldots, u_{p}(t)\right)$.

Для решения задачи (6.5)-(6.6) справедлива оценка

$$
\sum_{\alpha=1}^{p}\left\|u_{\alpha t}\right\|^{2}+\left\|\sum_{\alpha=1}^{p} A_{\alpha} u_{\alpha}\right\|^{2} \leqslant M\left(\|f\|^{2}+\left\|A u_{0}\right\|^{2}\right) .
$$

Из (6.7) следует непрерывная зависимость решения задачи (6.5)-(6.6) от входных данных в норме, стоящей в левой части оценки (6.7).

Каждую задачу (6.5)-(6.6) заменим разностной схемой

$$
\begin{gathered}
y_{\alpha t}+\sum_{\beta=1}^{\alpha} A_{\beta} \hat{y}_{\beta}+\sum_{\beta=\alpha+1}^{p} A_{\beta} y_{\beta}+\sum_{\beta=1}^{\alpha} A_{\beta} y_{\beta t}+\sum_{\beta=\alpha+1}^{p} A_{\beta} \stackrel{\vee v}{y t}_{\beta t}=f, \\
y_{\alpha}(0)=u_{0}, \quad \alpha=\overline{1, p} .
\end{gathered}
$$

Следует заметить, что задача (6.8)-(6.9) недоопределена. Для применения этой схемы требуется задать еще одно условие, например, значение $y_{\alpha}(t)$ на первом слое $y_{\alpha}(\tau)$. Будем искать $y(x, \tau)$ из соотношения

$$
\frac{u(x, \tau)-u_{0}(x)}{\tau}=\frac{\partial u}{\partial t}(x, 0)+O(\tau) .
$$

Значение $\left.\frac{\partial u}{\partial t}\right|_{t=0}$ ищем исходя из дифференциального уравнения (6.1)

$$
\left.\frac{\partial u}{\partial t}\right|_{t=0}=\sum_{\alpha=1}^{p} \frac{\partial}{\partial x_{\alpha}}\left(k_{\alpha}(x) \frac{\partial u_{0}(x)}{\partial x}\right)+\sum_{\alpha=1}^{p} \frac{\partial}{\partial x_{\alpha}}\left(k_{\alpha}(x) \frac{\partial}{\partial x_{\alpha}} u_{t}(x, 0)\right)+f(x, 0),
$$

или

$$
-\sum_{\alpha=1}^{p}\left(k_{\alpha}(x) u_{1 x_{\alpha}}\right)_{x_{\alpha}}+u_{1}(x)=F(x),\left.\quad u_{1}(x)\right|_{\Gamma}=0
$$

где

$$
F(x)=f(x, 0)+\sum_{\alpha=1}^{p}\left(k_{\alpha} u_{0 x_{\alpha}}\right)_{x_{\alpha}}, \quad u_{1}(x)=u_{t}(x, 0) .
$$

Итак, будем считать, что задача (6.8)-(6.9) дополнена еще одним условием

$$
y_{\alpha t}(0)=u_{1} \quad\left(y_{\alpha}(\tau)=u_{0}+\tau u_{1}=\bar{u}_{1}\right), \quad \alpha=\overline{1, p} .
$$

Применяя тот же прием, что и выше, получаем априорную оценку для решения разностной задачи (6.8)-(6.9), (6.11):

$$
\sum_{j^{\prime}=2}^{j}\left\|y_{\alpha t}^{j^{\prime}}\right\|^{2} \tau \leqslant M\left(\sum_{j^{\prime}=2}^{j}\left\|f_{t}^{j^{\prime}-1}\right\|^{2}+\sum_{\alpha=1}^{p}\left\|u_{1 \bar{x}_{\alpha}}\right\|^{2}+\left\|y_{1 t}^{0} f(\tau)-A \tilde{u}_{1}\right\|^{2}\right),
$$

где $\tilde{u}_{1}=(1+\tau) u_{1}+u_{0}, M-$ положительная постоянная, не зависящая от $h_{i}, i=\overline{1, p}, \tau$.

Из оценки (6.12) следует устойчивость разностной схемы (6.8)-(6.9), (6.11) по правой части и начальным данным. Из этой же оценки следует сходимость решения разностной задачи (6.8)-(6.9), (6.11) к решению дифференциальной задачи (6.1)-(6.2) со скоростью $O\left(|h|^{2}+\tau\right)$ при тех же условиях гладкости на $u(x, t)$, что и в теореме пункта 3.2.

Завышение гладкости $u(x, t)$ в рассматриваемых задачах объясняется присутствием в априорных оценках для погрешности $z_{\alpha}=y_{\alpha}-u_{\alpha}, \alpha=\overline{1, p}$, разностной производной от погрешности аппроксимации $\psi_{\bar{t}}^{j}$. 


\section{Литература}

1. Самарский А. А. Теория разностных схем.-М.: Наука, 1989.-161 с.

2. Марчук Г. И. Методы расщепления.-М.: Наука, 1989.-262 с.

3. Абрашин В. Н. Об одном варианте метода переменных направлений решения многомерных задач математической физики // Диф. уравнения.-1990.-Т. 26, № 2.-С. 314-323.

4. Абрашин В. Н., Муха В. А. Об одном классе экономичных разностных схем решения многомерных задач математической физики // Диф. уравнения.-1992.-Т. 28, № 10.-С. 1786-1799.

5. Абрашина-Жадаева Н. Г., Романова Н. С. Многокомпонентные векторные схемы расщепления для решения многомерных задач математической физики // Диф. уравнения.-2006. - Т. 42, № $7 .-$ C. $883-894$.

6. Вабищевич П. Н. Об одном классе векторных аддитивных схем // Изв. вузов. Математика.1994.-№ 9.-С. 11-15.

7. Самарский А. А., Гулин А. В. Устойчивость разностных схем.-М.: Наука, 1973.-415 с.

8. Вабищевич П. Н. Векторные аддитивные разностные схемы для эволюционных уравнений первого порядка // Журн. вычисл. математики и мат. физики.-1996.-Т. 36, № 3.-С. 44-51.

9. Самарский А. А., Вабищевич П. Н. Computational Modeling and Computing in Physics.-Dubna: JINR, 1997.-P. 363-371.

10. Самарский А. А., Вабищевич П. Н., Матус П. П. Устойчивость векторных аддитивных схем // Докл. РАН.-1998.-Т. 361, № 6.-С. 746-748.

11. Вабишевич П. Н. Регуляризованные аддитивные операторно-разностные схемы // Журн. вычисл. математики и мат. физики.-2010.-Т. 50, № 3.-С. 449-457.

12. Чудновский А. Ф. Теплофизика почвы.-М.: Наука, 1976. -352 с.

13. Виноградова М. Б., Руденко О. Б., Сухоруков А. П. Теория волн.-М.: Наука, 1979.-384 с.

14. Шхануков M. Х. Об одном методе решения краевых задач для уравнений третьего порядка // Докл. АН СССР.-1982.-Т. 265, № 6.-С. 1327-1330.

15. Солдатов А. П., Шхануков М. Х. Краевые задачи с общим нелокальным условием для псевдопараболических уравнений высокого порядка // Докл. АН СССР.-1987.-Т. 297, № 3.-С. 547-552.

Статъя поступила 1 июня 2011 г.

ШХАНУКОВ-ЛАФИШЕВ МУХАМЕД ХАБАЛОВИЧ

Кабардино-Балкарский госуниверситет им. Х. М. Бербекова,

заведующий кафедрой вычислительной математики

РОССИЯ, 360000, г. Нальчик, ул. Чернышевского, 165

E-mail: shkhanukov-lafishev@mail.ru

Архестова Сусанна Мухамедовна

Педагогический колледж Кабардино-Балкарского

госуниверситета им. Х. М. Бербекова,

преподаватель математики

РОССИЯ, 360000, г. Нальчик, ул. Чернышевского, 165

Тхамоков МУслим БАшиРОвич

Кабардино-Балкарский госуниверситет им. Х. М. Бербекова, старший преподаватель кафедры вычислительной математики РОССИЯ, 360000, г. Нальчик, ул. Чернышевского, 165

E-mail: kidmus@mail.ru

\section{VECTOR ADDITIVE SCHEMES FOR CERTAIN CLASSES OF HYPERBOLIC EQUATIONS}

\section{Shkhanukov-Lafishev M. H., Arhestova S. M., Tkhamokov M. B.}

Vector-additive schemes for certain classes of hyperbolic equations arising in the theories of moisture transport and waves in relaxing media are constructed. Stability and convergence of difference schemes in the class of sufficiently smooth solutions are proved.

Key words: wave equation, the equation of storage, stability, convergence, a priori estimate, viscosity, thermal conductivity, approximation error. 